\title{
BENJAMÍN GUTIÉRREZ: \\ PIONERO DE LA PRODUCCIÓN Y EL DESARROLLO DEL SAXOFÓN Y SU REPERTORIO CLÁSICO EN COSTA RICA
}

\author{
Benjamin Gutiérrez: Pioneer of the production and development \\ of saxophone and its classic repertoire in Costa Rica
}

Javier Valerio*

\begin{abstract}
RESUMEN
La Rapsodia para saxofón alto y orquesta de Benjamín Gutiérrez, representa un momento revolucionario para la historia de Costa Rica saxofón. Compositores costarricenses han escrito repertorio clásico importante a finales del siglo 20, contribuyendo así al desarrollo de la música de saxofón en general. Sin embargo, sus composiciones son poco conocidos fuera de sus círculos internos debido a la falta de ediciones musicales. A través de una serie de entrevistas y análisis este artículo refiere cómo Gutiérrez y la colaboración de Calderón cambiaron la forma de ver el saxofón de las elites musicales.

Definitivamente, hay más trabajo por hacer para el saxofón clásico en Costa Rica, ya que hay todavía una falta de publicaciones y grabaciones de estas composiciones. Además, un mayor desarrollo profesional de los saxofonistas y la pedagogía saxofón con sensibilidad cultural son esenciales para garantizar una información eficaz sobre la aparición y el desarrollo continuo del repertorio de saxofón clásico en Costa Rica.

Palabras clave: Saxofón, Benjamín Gutiérrez, Costa Rica, repertorio, composición musical.
\end{abstract}

\begin{abstract}
Benjamin Gutierrez's Rhapsody for Alto Saxophone and Orchestra represents a revolutionary moment for Costa Rican saxophone history. Costa Rica art composers have written an important classical repertoire in the late 20th century, thus contributing to the development of saxophone music in general. However, their compositions are little known outside of their inner circles due to the lack of music editions. Through a series of interviews and analyses this article portrays how Gutiérrez and Calderon's collaboration changed the way musical elitists view the saxophone.

There is definitely more work to be done for the classical saxophone in Costa Rica since there is still a lack of publications and recordings of these compositions. In addition, more professional development of saxophonists and culturally responsive saxophone pedagogy are essential to ensure efficient information about the continuous emergence and development of classical saxophone repertoire in Costa Rica.

Key Words: Saxofon, Benjamín Gutiérrez, Costa Rica, repertoire, musical composition.
\end{abstract}

Universidad de Costa Rica. Escuela de Artes Musicales. Costa Rica.

Correo electrónico: javier.valerio@ucr.ac.cr

Recepción: 12/5/16. Aceptación: 10/1/17. 
Compositores costarricenses han escrito una cuota significativa del repertorio para saxofón clásico a finales del siglo XX para contribuir con el desarrollo de la música para saxofón. Sus composiciones, sin embargo, son poco conocidas fuera de sus círculos internos musicales debido a la falta de publicaciones y porque trabajando en el aislamiento. La aparición de sus obras para saxofón, en la década de 1970, ha animado a otros compositores en el desarrollo de nuevas composiciones en varios géneros musicales y formatos, incluyendo obras para orquesta, banda, cámara y solo, así como música incidental y música producida con los medios electrónicos.

Los compositores costarricenses Benjamín Gutiérrez, Carlos Castro, Eddie Mora y Marvin Camacho influyeron, mediante sus composiciones, el repertorio de saxofón en Costa Rica. Gutiérrez escribió la Rapsodia para saxofón y orquesta en 1973; Castro escribió en 1989 Como un río, obra para saxofón alto y piano; Mora compuso en 1994 Diálogos, una obra de música de cámara para saxofón soprano y percusión múltiple. Asimismo, Camacho escribió Nocturno y Danza para saxofón solo en el 2011. Ellos fueron los primeros en componer obras de saxofón en Costa Rica y, como profesores de Composición, han tenido una gran influencia en las generaciones de compositores. Sus contribuciones han ayudado a poner el saxofón en un lugar de prominencia, a pesar de que el instrumento no fue muy respetado fuera de las Bandas Nacionales hasta la década de 1970 .

\section{Origen del saxofón en Costa Rica}

La historia del saxofón en Costa Rica se relaciona estrechamente con el desarrollo de las primeras instituciones de música en el país, tales como la Dirección General de Bandas, el Conservatorio de Castella, la Universidad de Costa Rica y el Instituto Nacional de Música.

Las bandas de concierto de Costa Rica se originaron como bandas militares, son los grupos musicales más antiguos, pues se remontan al año 1845 , cuando el Gobierno estableció el Departamento de Bandas ${ }^{1}$.

Las bandas militares, en cada región del país, estaban constituidas, principalmente, de instrumentos de metal y de percusión; más tarde, se convirtieron en ensambles completos incluyendo el saxofón. Las bandas acompañaban a las tropas en todas las actividades oficiales $\mathrm{y}$, además, proveían música en conciertos recreativos al aire libre; realizaban los conciertos los martes y los jueves por la noche en eventos conocidos como La retreta ${ }^{2}$. Los domingos por la mañana tocaban dos servicios: la Misa de Tropa, que era parte de la misa católica regular y el Recreo $^{3}$. La Misa de Tropa era acompañada por una banda militar en la que actuaba, como guardia de honor, el Presidente de la República. La Misa de Tropa sigue siendo una tradición que tiene muchos años de existencia, se originó en el antiguo régimen disciplinario del ejército, en la mitad del siglo XIX. El repertorio de las bandas incluyó marchas, valses, transcripciones de óperas y sinfonías.

La revolución de Costa Rica en 1948 trajo la abolición del ejército. Las funciones y las actividades de las bandas militares fueron redefinidas dentro de una esfera cultural y administrativa diferente y formaron parte de la Secretaría del Ministerio de Seguridad Pública, anteriormente conocido como Ministerio de Guerra y Marina.

Con la creación de la Dirección General de Bandas, en 1971, los instrumentalistas pudieron obtener un trabajo en el cual ejecutaban y enseñaban ${ }^{4}$. Muchos de los saxofonistas tocaban en ensambles de música clásica $\mathrm{y}$, también, en grupos de música popular bailable similares a las Big Bands de los Estados Unidos de Norteamérica; incorporaban instrumentos latinos (congas, timbales, bongos) y ritmos caribeños como el mambo y chachacha ${ }^{5}$. Sin embargo, aunque se permitía que el saxofón formara parte del formato de las bandas de concierto, este carecía del prestigio social de las elites musicales porque era un instrumento popular que tocaba música comercial como salsa, cumbia y merengue en las fiestas y en 
los salones de baile. Esta reputación negativa hizo que la enseñanza del instrumento fuera todavía más difícil de ser aceptada en los programas curriculares de las instituciones musicales existentes ${ }^{6}$.

Varios años después, debido al interés de los seguidores de las bandas, las nuevas generaciones y la razón de que compositores como Benjamín Gutiérrez ocuparan puestos administrativos en las instituciones musicales, hizo que el saxofón fuera, finalmente, incluido en los programas curriculares del Conservatorio de Castella, de la Universidad de Costa Rica y del Instituto Nacional de Música. Estas entidades ofrecían clases individuales desde el nivel elemental hasta el universitario y estaban disponibles para el público en general. Inicialmente, el Conservatorio de Castella, en los años setenta y ochenta, contrataba músicos de la Banda Nacional de San José como profesores de instrumento para enseñar a los nuevos estudiantes.

El creciente número de estudiantes de saxofón, a su vez, permitió a la Universidad de Costa Rica, a la Universidad Nacional y al Instituto Nacional de Música incorporar este instrumento en los currículos musicales a mediados de los años ochenta y ofrecer el grado de Bachillerato. Los profesores desarrollaron métodos de enseñanza adecuados para el repertorio del saxofón clásico, el que, más tarde, fue utilizado en otras instituciones musicales.

\section{Benjamín Gutiérrez}

Benjamín Gutiérrez es, sin duda, el compositor más importante en Costa Rica. Nació en 1937 e ingresó al Conservatorio de Música de la Universidad de Costa Rica en 1953 para estudiar piano. En 1957, se marchó a Guatemala, en donde estudió piano y composición en el Conservatorio Nacional de Música. Un año más tarde, inició sus estudios de Maestría en Composición en el programa de música del Conservatorio de New England, en Boston, Massachusetts. Estudió composición con maestros como Francis Judd Cooken y Carl McKinley.

Gutiérrez recibió una beca en el Conservatorio para participar en el Festival de Música de Casals, en Puerto Rico, y en el Festival de Música de Aspen, en Colorado. La experiencia de Aspen tuvo un gran impacto en la carrera musical de Gutiérrez porque allí se encontró con el compositor francés Darius Milhaud (1892-1974), quien fue instructor en el Festival. Gutiérrez recibió un valioso consejo de Milhaud:

Tuve la suerte suficiente de conocer a Darius Milhaud cuando fui a Aspen. Él conocía América Latina debido a los años que pasó en Brasil como agregado Cultural en la Embajada de Francia. Me dijo que siempre debo recordar que era de América Latina. Que debo aplicar todas las técnicas aprendidas en los Estados Unidos a lo que me gustaba y era inherente a mis raíces. Esto era muy importante para mí porque me convertí en un defensor de este principio ${ }^{7}$.

Milhaud alentó a Gutiérrez para componer música en un lenguaje tonal, a diferencia de lo que sus colegas hacían en el Festival. Con la supervisión de Milhaud, compuso una de sus obras para piano solo, la Tocata y Fuga ${ }^{8}$ y recibió una mención honorífica en la competencia del festival estudiantil de verano9.

Gutiérrez terminó su maestría y regresó a Costa Rica en 1960. Luego, el Decano de la Facultad de Educación de la Universidad de Costa Rica le aconsejó estudiar Educación Musical y, en 1961, se inscribió en el programa de Maestría de Educación Musical en la Universidad de Michigan. Estudió composición como segundo énfasis con Ross Lee Finney (1906-1997), exalumno de Nadia Boulanger (1887-1979), Alban Berg (1885-1935) y Roger Sessions (1896-1985). Se graduó en 1962 y regresó a su país natal para enseñar en la Universidad de Costa Rica, Facultad de Educación. Un año más tarde, se convirtió en el director y uno de los fundadores de la Escuela de Música de la Universidad de Costa Rica.

En 1965, recibió una beca para estudiar con Alberto Ginastera en el Centro Latinoamericano de Estudios Musicales del 
Instituto Torcuato Di Tella, en Buenos Aires, Argentina. Gutiérrez describió a Ginastera como un profesor exigente, quien esperaba que los estudiantes estuvieran absolutamente comprometidos con su carrera de composición. Aunque animó a sus estudiantes a explorar los estilos que más les inclinaban, se esperaba que los estudiantes del Instituto se enfocaran en las técnicas de vanguardia que estaban en práctica ${ }^{10}$.

Gutiérrez estudió orquestación y composición con Ginastera, quien tuvo un papel importante en su formación artística y estilo musical. Gutiérrez estaba profundamente interesado en la música latinoamericana y música atonal de igual forma. También, tuvo contacto con compositores prominentes del siglo XX, como Olivier Messiaen, Iannis Xenakis, Luigi Nono, Aaron Copland y Luigi Dallapiccola, quienes visitaron el Centro Latinoamericano de Música ${ }^{11}$.

Gutiérrez construyó su reputación como compositor en las décadas de 1960 y 1970, y continuó produciendo obras importantes a mediados de 1980. En 1984 viajó a París y asistió a un seminario sobre la música electrónica en el IRCAM (Institut de Recherche et Coordination Acoustique/Musique), bajo la dirección de Pierre Boulez. En general, Gutiérrez no se considera un seguidor de un estilo en particular. Admiraba el lirismo de Brahms, que incorporó en sus obras. Su orquestación utiliza diversos lenguajes armónicos sin abandonar la tonalidad, aunque algunas de sus obras están escritas con un lenguaje politonal.

Desde 1994, Gutiérrez fue el enlace de Costa Rica para un programa que otorgaba becas a los costarricenses para estudiar Música en Francia. Asimismo, ha dirigido orquestas, coros y óperas en Latinoamérica y en Europa. Ha recibido importantes premios, reconocimientos y homenajes de la Alpha Lotta Association -1959-, Juegos Florales de Guatemala -1966-, composición Premio Aquileo Echeverría ${ }^{12}$-1962-64, 1973, 1977, 1980 y 1985 - y el Premio Nacional de Música -1973, 1977, 1980 y 1985-, Premio de Música de cámara, Teatro Nacional de Costa Rica
1978. También, fue declarado compositor del siglo XX por el periódico La Nación en 1999, y Premio Nacional de Cultura Magón ${ }^{13}$.

\section{A. Benjamín Gutiérrez, Rapsodia para saxofón y orquesta (1977)}

En 1973, Gutiérrez compuso la Rapsodia para saxofón alto y orquesta y se la dedicó a Norman Calderón, talentoso saxofonista autodidacto costarricense quien fue en busca de nuevo repertorio para saxofón clásico. En la década de 1970 no había todavía una tradición o educación formal para el saxofón clásico en Costa Rica; además, había poca información sobre el repertorio clásico del saxofón en Europa y en los Estados Unidos de Norteamérica. Aunque Calderón quería tocar el saxofón, para entrar en el Conservatorio de Música tuvo que elegir entre el clarinete o el oboe.

En ese período, el saxofón era considerado un instrumento inferior, conveniente solamente para música popular. Calderón trabajó en la Banda Nacional de Conciertos de San José, donde solía ejecutar transcripciones para saxofón de óperas italianas y fantasías originalmente compuestas para oboe y clarinete. Estas transcripciones llamaron la atención de músicos, quienes vieron posibilidades para el desarrollo de un repertorio clásico de saxofón.

En 1972, Calderón obtuvo un catálogo de la editorial francesa Alphonse Leduc y notó una cantidad impresionante de obras originales para saxofón clásico. Al azar seleccionó el Concertino da Cámara para saxofón alto y 11 instrumentos de Jacques Ibert (1890-1962) y pidió a Gutiérrez -quien, en aquel momento, era el director del Conservatorio de Música y director de la Orquesta Sinfónica- para realizar la pieza. Calderón recuerda ese momento:

Fue muy difícil para mí estar en esta posición. Muchos amigos no apoyaban mi idea de tocar un concierto ya que el saxofón no era considerado un instrumento serio. Recuerdo lo nervioso que estaba cuando finalmente realicé esta obra en el Teatro Nacional. Después del concierto, las personas me respetaron más y me sentí bien acerca de lo que había hecho para el instrumento ${ }^{14}$. 
Desde la infancia, Gutiérrez tenía una admiración especial para el saxofón porque creció escuchando los conciertos de la bandas en Costa Rica ${ }^{15}$. Fue después de la ejecución de Calderón del Concertino da cámara que decidió componer una obra para saxofón basado en su anterior concierto para clarinete y orquesta (1960). En 1974, Gutiérrez terminó su Rapsodia para saxofón alto y orquesta y, además, la transcripción para piano en caso de que una orquesta sinfónica no fuera disponible. Rapsodia para saxofón alto y orquesta representa un momento revolucionario para la historia del saxofón de Costa Rica. El trabajo en equipo de Gutiérrez y de Calderón cambió la reputación del saxofón dentro del ambiente elitista musical. Posteriormente, otras instituciones de la música incorporan el saxofón dentro de sus programas, alentando a compositores y saxofonistas para promover el repertorio clásico de este instrumento.

\section{B. Análisis de la obra}

La Rapsodia de Gutiérrez se compone de tres movimientos: Allegro con fuoco, Andante moderato y Allegro marcatto, cada uno muy expresivo y con yuxtaposiciones atrevidas de estilos y las secciones contrastantes. El resultado es un trabajo rico en su textura de considerable variedad y profundidad adaptado maravillosamente al piano y al saxofón. Hablando del título y de la estructura general de la obra, las notas del compositor:

Le llamé Rapsodia debido a sus tres elementos primarios o estilos. La primera es una mezcla de lirismo romántico y post-romanticismo, una especie de homenaje al siglo XIX. El segundo movimiento es dodecafónico [es decir, serial] y el tercero es cuasi-tonal en virtud de basarse en algún material de un concierto de Bach $^{16}$.

El primer movimiento comienza con una animosa introducción que incluye los dos temas principales, como se indica en los ejemplos la y 1b. Ambos comienzan con una negra con punto seguido por una corchea, que crea la sensación de continuidad motívica que equilibra muy bien la ambigüedad tonal, tan a menudo presente en la obra y que será discutido más adelante.

Dada la presencia de estos dos temas claramente definidos, su desarrollo considerable en todo el movimiento y el retorno audaz del primer tema en compas 126 , se considera a la obra como una forma de sonata libremente construida; es una herramienta interpretativa útil. Sin embargo, y esto es más acorde con su carácter rapsódico, el movimiento puede ser visto en términos de una simple forma de arco, con el clímax sucediendo cerca de la tercera parte del movimiento. Como se señaló anteriormente, el lenguaje armónico es complejo y es descrito por el propio compositor como politonal, aunque esto solo es cierto en el sentido más amplio de la palabra ${ }^{17}$.

Por ejemplo, la última parte de la introducción, en los compases 15-22, establece claramente el V a I resolviendo en F \# Mayor en el piano en el compás 23. Sin embargo, también es la primera entrada esperada del saxofón, cuyo centro tonal inicial es $\mathrm{C}$, la máxima distancia armónica del tritono lejos de la fundamental, $\mathrm{F} \#$, en el piano. Aun así, este choque de tonalidades no persiste, ya que la línea del saxofón retorna rápidamente a una órbita libre alrededor $\mathrm{F}$ \# mayor (ver ejemplo 2, abajo). De hecho, Gutiérrez parece elegir acordes basados en su sonoridad como de cualquier consideración sistemática, intercalando tritonos, sétimas mayores y segundas menores, pero manteniendo un enfoque esencialmente triádico en todo.

Ejemplo 1a. y 1b. (compases 23-26 y 64-65, respectivamente, escrito abajo en tono de concierto).

a)

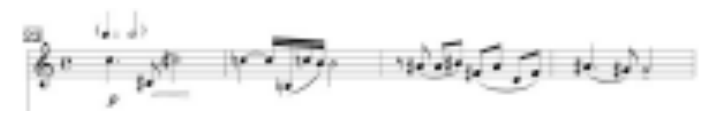

b)

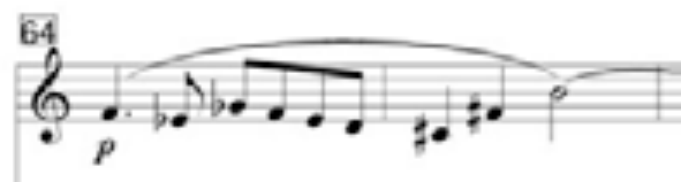


Ejemplo 1c. (compases 17-26, saxofón en tono de concierto y piano):

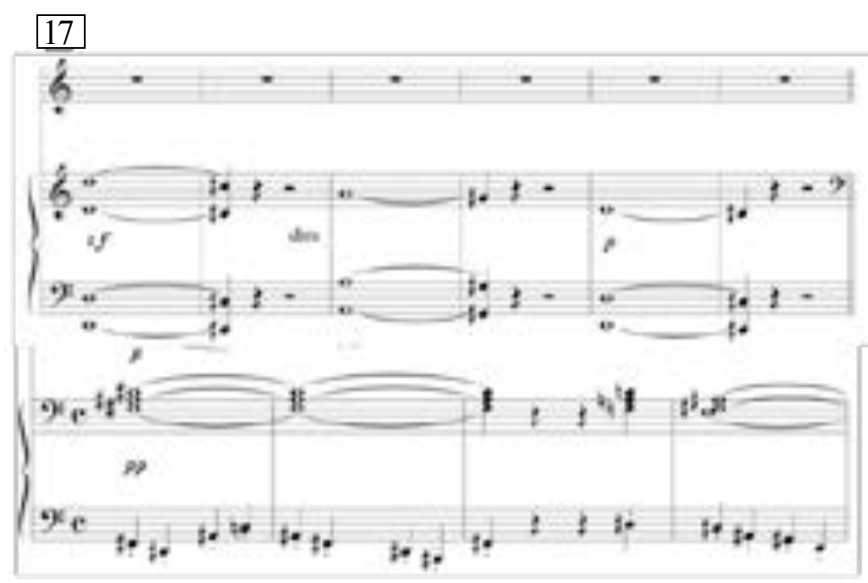

Por último, vale la pena mencionar que la escritura melódica del compositor, en este movimiento, son reminiscencias del
Romanticismo, en donde las melodías, a menudo, alcanzan su cumbre hacia el final de la línea.

Ejemplo 1d. (compases 86-89 partitura del saxofón):
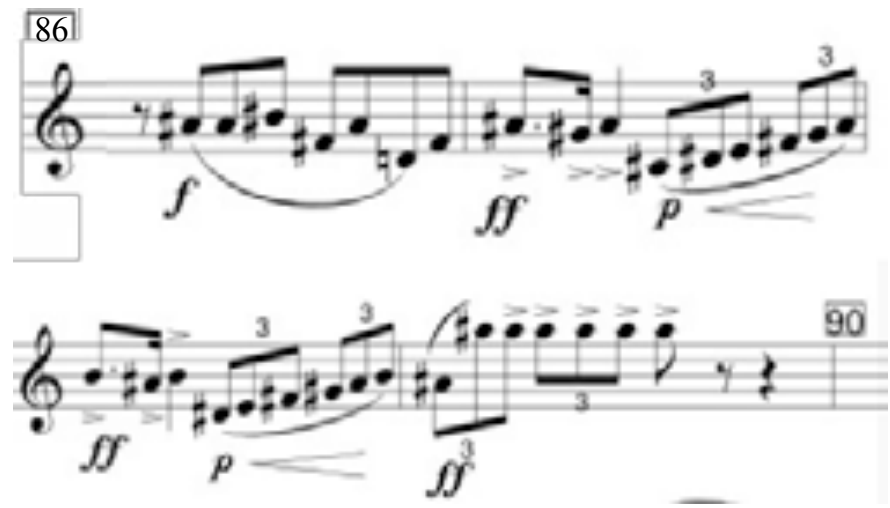

Gutiérrez describió el segundo movimiento como un "dodecafonismo lírico", teniendo en cuenta el carácter global de la música y su organización dodecafónica subyacente. Sin embargo, a diferencia de la poli-tonalidad libre del primer movimiento, se aplica la técnica serial de una manera muy libre en todo el movimiento.
Mientras que el espacio no permite un análisis completo de la pieza, algunas observaciones serán suficientes aquí para observar la forma y la organización dodecafónica básica del movimiento. A continuación, una matriz de doce tonos para la pieza, usando el método del oído absoluto, donde el principio de la fila de do es P0: 


\begin{tabular}{|c|c|c|c|c|c|c|c|c|c|c|c|c|c|}
\hline & $I_{10}$ & $1_{4}$ & 19 & 18 & $1_{7}$ & $1_{0}$ & $1_{4}$ & $I_{5}$ & $I_{11}$ & $I_{6}$ & $\mathrm{I}_{2}$ & $I_{1}$ & \\
\hline & 86 & $E$ & A & $A B$ & G & c & 00 & $F$ & B & $F=$ & D & $E D$ & $\mathbb{R}_{1}$ \\
\hline$P_{4}$ & $E$ & 80 & $E D$ & D & 00 & Fat & G & B & $F$ & C & $A D$ & A & $\mathbf{R}_{4}$ \\
\hline$P_{11}$ & B & $F$ & $\mathrm{Bb}$ & A & $A B$ & $\mathrm{DO}$ & D & Fill & C & G & $E D$ & $\mathrm{E}$ & $R_{11}$ \\
\hline $\mathbf{P}_{0}$ & C & F" & B & $B b$ & A & $D$ & $E b$ & 0 & $\mathrm{Db}$ & $A b$ & 2 & $F$ & $R_{0}$ \\
\hline$P_{1}$ & $D b$ & G & C & B & $B b$ & $E b$ & $E$ & $A b$ & D & A & 1 & $\mathrm{~F}=$ & $R_{1}$ \\
\hline $\mathrm{P}_{\mathrm{s}}$ & $\mathrm{Ab}$ & D & G & $\mathrm{Fa}$ & $F$ & $B b$ & B & $E b$ & A & E & C & $D b$ & $R_{S}$ \\
\hline $\mathrm{P}_{7}$ & $G$ & Do & Fu & $F$ & $\mathrm{E}$ & A & $B b$ & D & $A b$ & ED & B & $\mathrm{c}$ & $\mathrm{R}_{7}$ \\
\hline $\mathbf{P}_{3}$ & $E D$ & A & D & Do & c & $F$ & $\mathrm{FuH}$ & $B 6$ & $E$ & B & $G$ & $A D$ & $R_{3}$ \\
\hline $\mathrm{P}_{9}$ & A & $E D$ & AD & $G$ & $\mathrm{~F} \#$ & B & c & $\mathrm{E}$ & 86 & $F$ & 00 & D & $\mathbb{R}_{9}$ \\
\hline $\mathrm{P}_{2}$ & D & AD & Do & c & B & $\mathbf{E}$ & $F$ & A & $E D$ & $B b$ & $\mathrm{~F}=$ & $G$ & $\mathrm{R}_{2}$ \\
\hline$P_{6}$ & $\mathrm{Fat}$ & C & $F$ & E & $E B$ & $A B$ & A & Do & G & D & $B b$ & B & $\mathbb{R}_{6}$ \\
\hline$P_{5}$ & $F$ & B & $\mathrm{E}$ & $E \mathrm{ED}$ & D & G & $A b$ & c & $F^{w}$ & Do & A & $\mathrm{Bb}$ & $R_{s}$ \\
\hline & & & & & RI- & $\mathrm{RL}_{3}$ & $\mathrm{RI}_{1}$ & & & & & $R t$ & \\
\hline
\end{tabular}

La serie dodecafónica inicial, P10, se encuentra en el lado izquierdo desde el primer compás 1 hasta el segundo tiempo del segundo compás. Esta fila y sus transposiciones son el "adhesivo principal de la serie" que une las ideas que aparecen en la línea del bajo a lo largo (compases 1, 14, 20, 32). Curiosamente, la propia melodía del saxofón, mientras hace el ciclo rápidamente por todas las doce notas, aunque con algunas repeticiones, no se ajusta exactamente a ninguna de las filas dadas anteriormente. En su lugar, sirve como una especie de "rumia obligato" en el material presentado, más literalmente en el piano (ver a continuación el ejemplo 2a).

Ejemplo 2a (compases 1-6):

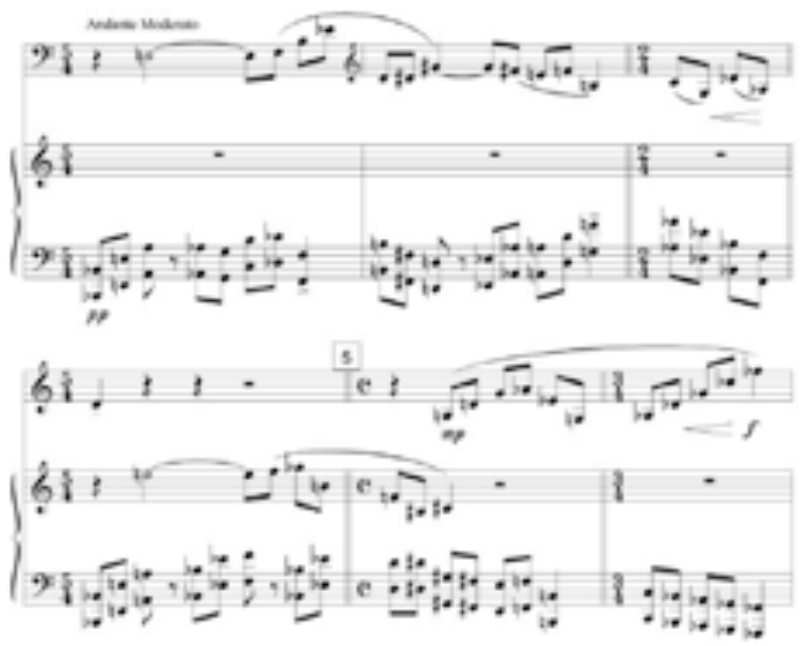


El movimiento es, en gran parte, compuesto y unido por los elementos unificadores que acabamos de mencionar. Además, un motivo ascendente, tomado del primer intervalo de la línea del saxofón -véase el ejemplo 1c anterior-

Ejemplo 2b (compás 20)

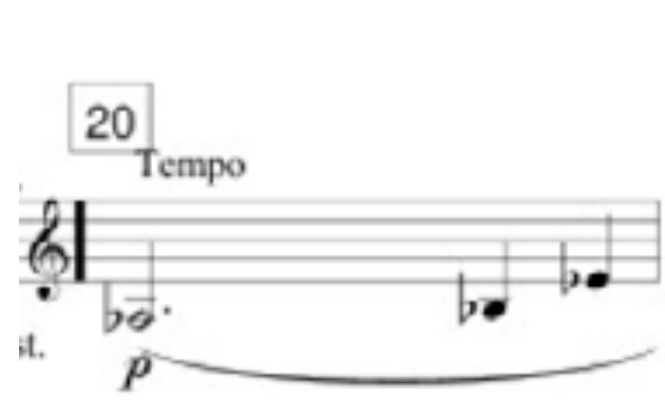

El tercer movimiento, Gutiérrez lo describe como un "Homenaje a la N. ${ }^{\circ} 1$ de Johann Sebastian Bach, en re menor, BWV 1052. Este movimiento, que sigue el movimiento anterior sin pausa, comienza con material tomado de la en la parte saxofón vuelve, en repetidas ocasiones, como una especie de meditación en ascenso, como se ha indicado en el ejemplo $2 \mathrm{~b}$ y $2 \mathrm{c}$ que se presenta a continuación.

Ejemplo2c (compás 32)

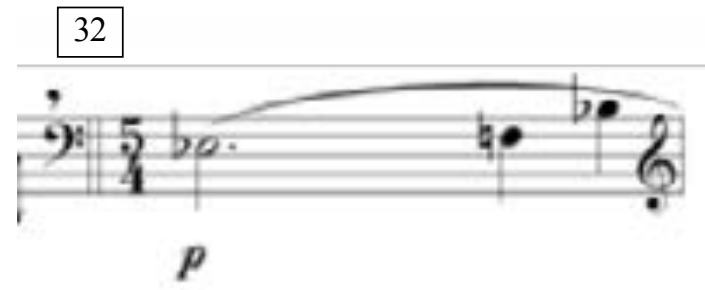

introducción del primer movimiento y reproduce la tonalidad de do \# menor y, junto con otras referencias a los dos primeros movimientos, para dar un carácter cíclico a toda la Rapsodia (véase el ejemplo 3a).

Ejemplo 3a (compases. 1- 7):

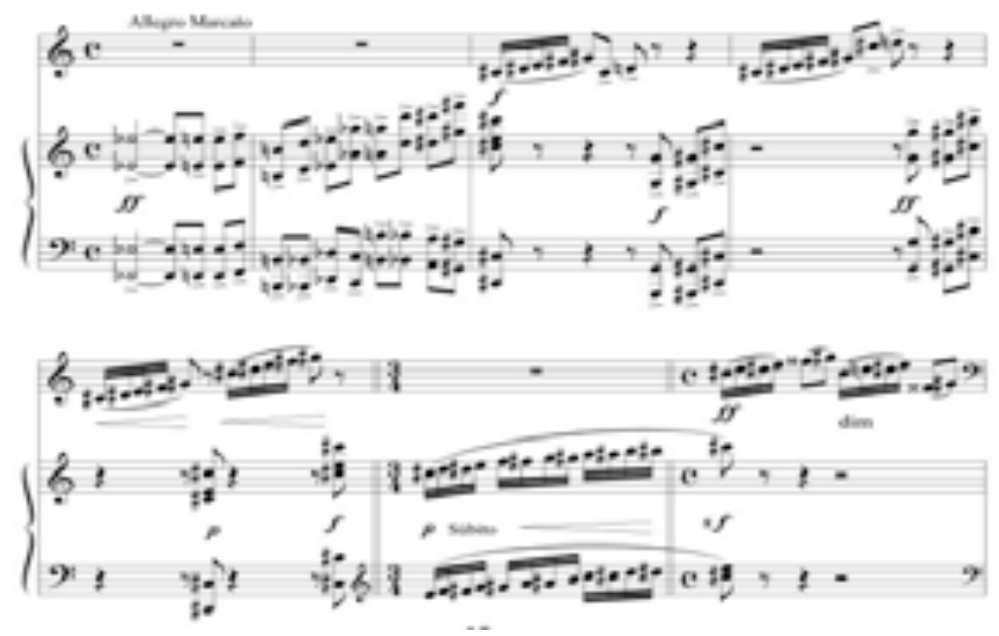


El tema se presenta al iniciar en el compás $3 \mathrm{y}$, posteriormente, se desarrolla motivicamente en centros tonales alternados de do \# y fa \# (véase el ejemplo 3b). El ritmo y la métrica sobresalen mucho más en este movimiento que en los dos anteriores, con una variedad mucho más rítmica, hasta el punto de ser discordante, aunque todo dentro de una métrica de $4 / 4$. (véase el ejemplo 3c).

Ejemplo 3b:

i. Compases $87-88$

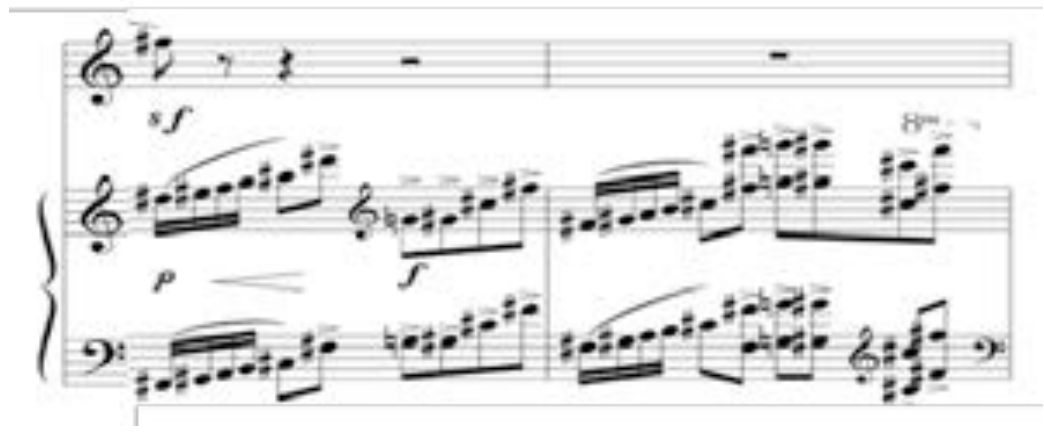

ii. Desde la cadenza (compás. 104)

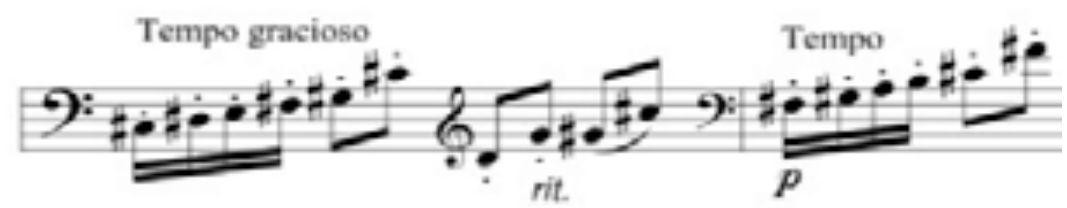

Ejemplo 3c:

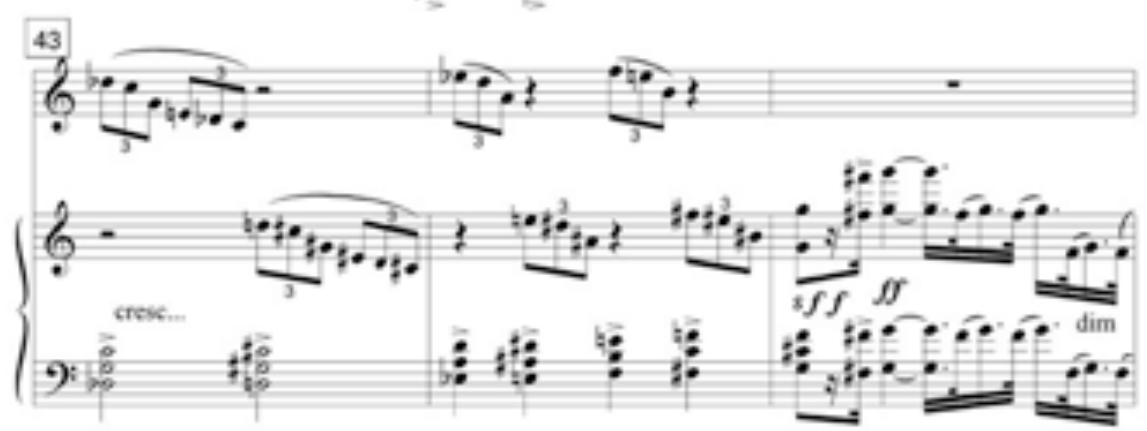


La estructura formal se define más claramente en este movimiento, con más de un gesto de la forma del Ritornello barroco (véase el ejemplo 3d) que se encuentra, a menudo, en los conciertos de esa época. Por otra parte, los movimientos cuentan con un gran contraste de estados de ánimo entre el saxofón y la orquesta. Estos sirven para acentuar la versatilidad del sonido del saxofón.

Ejemplo 3d:

i. Compases 3-4 (presentación inicial)

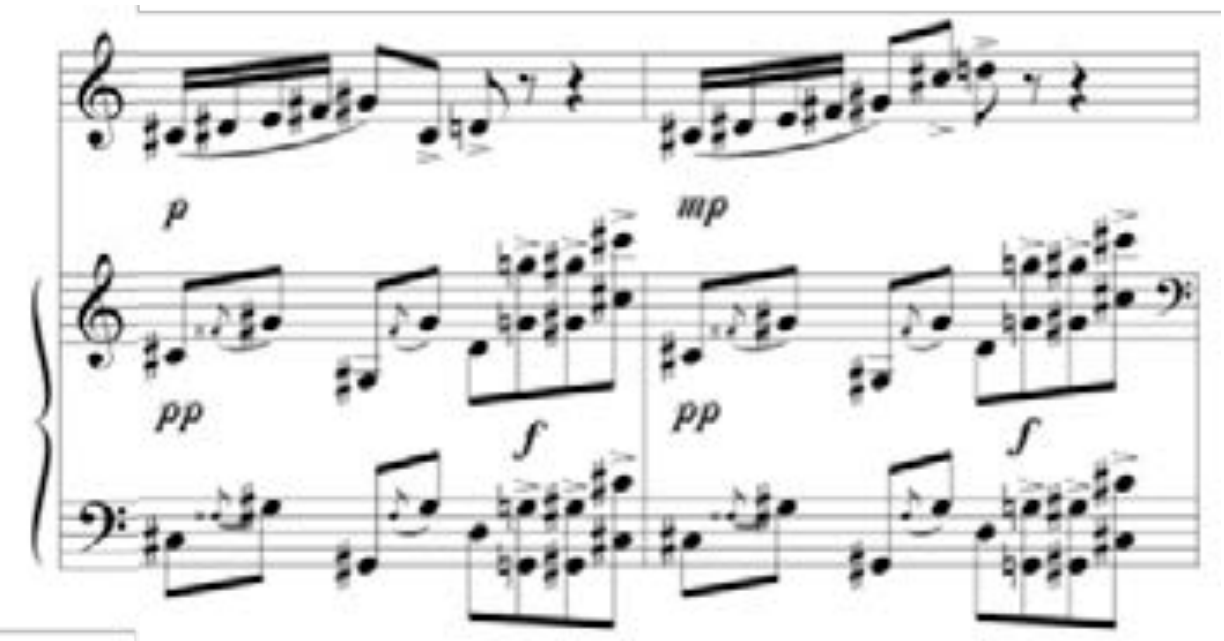

ii. Compases 14-15 Ritornello (modificado)

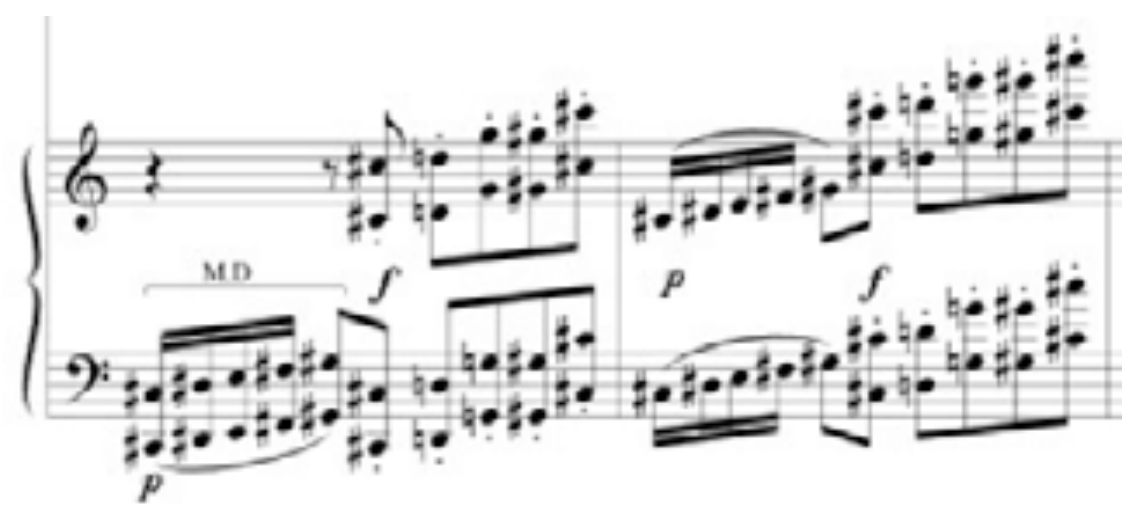




\section{Conclusión}

Gutiérrez no solo sentó las bases de un repertorio de saxofón clásico en Costa Rica, inspirando a nuevas generaciones de compositores como Carlos Castro, Eddie Mora y Marvin Camacho, sino que, gracias a sus posiciones administrativas, también logró asentar la creación del programa de saxofón en instituciones musicales de Costa Rica.

Actualmente, los estudiantes tienen la oportunidad de obtener un título de la Universidad de Costa Rica, la Universidad Nacional o el Instituto Nacional de Música y convertirse en saxofonistas profesionales. Desde el año 2007, con el fin de ampliar la cultura musical de la gran Área Metropolitana y proporcionar acceso a la educación de la Música a la gente en otras zonas del país, el Gobierno de Costa Rica y el Ministerio de Cultura crearon el SINEM (Sistema Nacional de Escuelas de Música). Estas escuelas han proporcionado más oportunidades para los instructores de saxofón con el fin de enseñar el instrumento en comunidades remotas, en donde la instrucción antes no era accesible.

También, en 2007 el país comenzó a organizar el Sax Fest Costa Rica Internacional, evento en el cual los métodos pedagógicos y el repertorio del saxofón pueden ser discutidos y las actuaciones de los saxofonistas, en toda América Latina, pueden ser presentadas.

Las composiciones de saxofón de Gutiérrez, Castro, Mora y Camacho también han inspirado a compositores jóvenes costarricenses, entre ellos: Allen Torres, Vinicio Meza, Nelson Ramírez y Otto Castro, para escribir nuevas obras para el saxofón. La mayoría de estos compositores ya no funciona de manera aislada, dado que, ahora, son miembros de ACAM (Asociación de Compositores y Autores Musicales de Costa Rica). Sus composiciones han sido interpretadas en festivales de música y congresos en todo el mundo, lo que ha permitido a otros músicos aprender acerca del repertorio de saxofón en Costa Rica.

Definitivamente, hay más trabajo por hacer para el saxofón clásico en Costa Rica, ya que, todavía, hacen falta publicaciones y grabaciones de estas composiciones. Además, los métodos pedagógicos y el desarrollo profesional de los músicos son esenciales para garantizar el continuo desarrollo del repertorio de saxofón clásico en Costa Rica.

\section{Notas}

1. Pompilio Segura Chaves (2001). Desarrollo musical en Costa Rica durante el siglo XIX: las bandas militares Heredia, Costa Rica: EUNA.

2. Retreta: conciertos al aire libre generalmente interpretados por bandas de concierto en parques de la ciudad y por la noche.

3. Conciertos dominicales al aire libre, generalmente interpretados por bandas de concierto en parques de la ciudad durante la mañana.

4. Molina-Jiménez, Iván. (2003). Identidad nacional y cambio cultural en Costa Rica durante la segunda mitad del siglo XX. San José: Editorial de la Universidad de Costa Rica.

5. Ídem.

6. Flores, Bernal. (1978). La música en Costa Rica. San José: Editorial Costa Rica.

7. Benjamín Gutiérrez, entrevista telefónica con el autor, 18 noviembre de 2010.

8. Jorge Carmona Ruiz. (2008). Sonatas para piano de construcción serialista en Centroamérica en la segunda mitad del siglo XX. Heredia: Universidad de Costa Rica.

9. Benjamín Gutiérrez, entrevista por Juan Pablo Andrade, 5 de marzo de 2007.

10. Benjamín Gutiérrez, entrevista con el autor el 18 de noviembre de 2010.

11. Benjamín Gutiérrez, entrevista por Juan Pablo Andrade, el 5 marzo de 2007.

12. Aquileo J. Echeverría (1866-1909), poeta costarricense, periodista y diplomático. 
13. Benjamín Gutiérrez, entrevista por Juan Pablo Andrade, el 5 marzo de 2007.

14. Norman Calderón. Entrevista telefónica por el autor el 20 de noviembre de 2010.

15. Norman Calderón. Entrevista telefónica por el autor el 20 de noviembre de 2010.

16. Ídem.

17. Benjamín Gutiérrez. Entrevista realizada por el autor el 18 de noviembre de 2010 .

\section{Bibliografía}

Andrade, Juan Pablo. 2007, 5 Mar. Entrevista con Benjamín Gutiérrez.

Arguedas, Iván. 2010. De la evolución del saxofón y su llegada a la academia: tres perspectivas con reverberación costarricense. San José, C.R.: Escuela de Artes Musicales de la UCR.

Barquero, Zamira \& Cabezas, Esteban. 2002. Catálogo de manuscritos e impresos del Archivo Histórico Musical. San José, C.R: Escuela de Artes Musicales de la UCR.

Calderón, Norman. 2010, 27 Octubre. Entrevista personal.

Calderón, Norman. 2010, 20 Noviembre. Entrevista personal.

Flores, Bernal. 1978. La Música en Costa Rica. San José: Editorial Costa Rica.

Helfengerber, Krista. 2009. "Sonata for Clarinet and Piano by Benjamín Gutiérrez: contribution to Costa Rican musical heritage". Arts Scene Magazine. (65): 27-34.
García, Fernando. 1998. Saxofón en concierto. Compositores chilenos. Santiago, Chile.

Gutiérrez, Benjamín. 2010, 20 Noviembre. Entrevista personal

Gutiérrez, Benjamín. 2012, 18 Diciembre. Entrevista personal.

Londeix, Jean-Marie. 1994. 150 Years of Music for Saxophone: Bibliographical Index of Music and Educational Literature for the Saxophone, 1844-1994. Cherry Hill, NJ: Roncorp.

Segura Chaves, Pompilio. 2001. Desarrollo musical en Costa Rica durante el siglo XIX: las bandas militares. Heredia, Costa Rica: EUNA.

Sider, Ronald. 1984. Comtemporary composer in Costa Rica. Latin American Music Review / Revista de Música Latinoamericana. 5 (2), Autumn-Winter. University of Texas Press.

Slonimsky, Nicolas. 1945. Music of Latin America. New York: Thomas Y. Crowell Company.

Vargas Cullell, María Clara. 2004. De las fanfarrias a las salas de concierto: música en Costa Rica (1840-1940). San José, Costa Rica: Editorial de la Universidad de Costa Rica.

Vargas Cullell, María Clara. 2012. "Un escenario caleidoscópico: Música en Costa Rica (1940-2010)". En: María Clara Vargas Cullell, Ekaterina Chatski \& Tania Vicente. Música académica costarricense. Del presente al pasado cercano. San José: Facultad de Bellas Artes de la Universidad de Costa Rica, 19- 58. 
Vicente, Tania. 2009. Bibliografías de compositores contemporáneos. San José, Costa Rica: Universidad de Costa Rica.

Villafruela, Miguel. 2007. El saxofón en la música Latinoamericana. Santiago de Chile: Universidad de Chile.
Villafruela, Miguel. (1999). El saxofón y los compositores latinoamericanos. Catalogación de obras escritas para el saxofón. Habana, Cuba.

Zaldívar, Mario. 2003. Imágenes de la música popular costarricense, 1939-1965. San José: Editorial de la Universidad de Costa Rica.

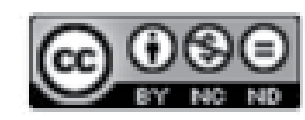

Este obra está bajo una licencia de Creative Commons

Reconocimiento-NoComercial-SinObraDerivada 4.0 Internacional. 